\title{
An implicit difference scheme with the KPS preconditioner for two-dimensional time-space fractional convection-diffusion equations ${ }^{\text {th }}$
}

\author{
Yongtao Zhou $^{\mathrm{a}, \mathrm{b}}$, Chengjian Zhang, ${ }^{\mathrm{a}, \mathrm{b}, *}$, Luigi Brugnano ${ }^{\mathrm{c}}$ \\ ${ }^{a}$ School of Mathematics and Statistics, Huazhong University of Science and Technology, Wuhan 430074, China \\ ${ }^{b}$ Hubei Key Laboratory of Engineering Modeling and Scientific Computing, Huazhong University of Science and Technology, \\ Wuhan 430074, China \\ ${ }^{c}$ Dipartimento di Matematica e Informatica "U. Dini", Università di Firenze, Viale Morgagni 67/A, Firenze I-50134, Italy
}

\begin{abstract}
This paper deals with the numerical computation and analysis for a class of two-dimensional time-space fractional convection-diffusion equations. An implicit difference scheme is derived for solving this class of equations. It is proved under some suitable conditions that the derived difference scheme is stable and convergent. Moreover, the convergence orders of the scheme in time and space are also given. In order to accelerate the convergence rate, by combining the Kronecker product splitting (KPS) preconditioner with the generalized minimal residual (GMRES) method, a preconditioning strategy for implementing the difference scheme is introduced. Finally, several numerical examples are presented to illustrate the computational accuracy and efficiency of the methods.
\end{abstract}

Keywords: Fractional convection-diffusion equations, Implicit difference scheme, Stability, Convergence, Kronecker product splitting preconditioner

\section{Introduction}

Consider the following initial-boundary problems of two-dimensional time-space fractional convectiondiffusion equations:

$$
\left\{\begin{array}{l}
a_{1} \frac{\partial u(x, y, t)}{\partial t}+a_{2}{ }_{t_{0}}^{C} D_{t}^{\gamma} u(x, y, t) \\
=a_{3} \frac{\partial u(x, y, t)}{\partial x}+a_{4} \frac{\partial u(x, y, t)}{\partial y}+a_{5} \frac{\partial^{\alpha} u(x, y, t)}{\partial|x|^{\alpha}}+a_{6} \frac{\partial^{\beta} u(x, y, t)}{\partial|y|^{\beta}}+f(x, y, t), \quad(x, y, t) \in \Omega \times\left(t_{0}, T\right], \\
u\left(x, y, t_{0}\right)=u_{0}(x, y), \quad(x, y) \in \bar{\Omega}=\Omega \cup \partial \Omega ; u(x, y, t)=0, \quad(x, y, t) \in \mathbb{R}^{2} \backslash \Omega \times\left[t_{0}, T\right],
\end{array}\right.
$$

where $a_{i}(i=1,2, \ldots, 6), \alpha, \beta$ and $\gamma$ are some given constants with $a_{1}, a_{2}, a_{5}, a_{6} \geq 0,\left|a_{1}\right|+\left|a_{2}\right| \neq 0$, $\left|a_{5}\right|+\left|a_{6}\right| \neq 0,1<\alpha, \beta \leq 2$ and $0<\gamma<1, \Omega=\left(x_{L}, x_{R}\right) \times\left(y_{L}, y_{R}\right)$ denotes the space domain with boundary $\partial \Omega, f(x, y, t)$ is the source term, ${ }_{t_{0}}^{C} D_{t}^{\gamma} u(x, y, t)$ is the $\gamma$-order Caputo fractional derivative of unknown function $u(x, y, t)$ defined by

$$
{ }_{t_{0}}^{C} D_{t}^{\gamma} u(x, y, t)=\frac{1}{\Gamma(1-\gamma)} \int_{t_{0}}^{t} \frac{\partial u(x, y, \xi)}{\partial \xi} \frac{d \xi}{(t-\xi)^{\gamma}},
$$

\footnotetext{
This work is supported by NSFC (Grant No. 11971010).

* Corresponding author

Email addresses: yongtaozh@126.com (Yongtao Zhou), cjzhang@mail.hust.edu.cn (Chengjian Zhang), luigi.brugnano@unifi.it (Luigi Brugnano)
} 
$\frac{\partial^{\alpha} u(x, y, t)}{\partial|x|^{\alpha}}$ is the $\alpha$-order Riesz fractional derivative of $u(x, y, t)$ w.r.t. the variable $x$ defined by

$$
\frac{\partial^{\alpha} u(x, y, t)}{\partial|x|^{\alpha}}=-\frac{1}{2 \cos \left(\frac{\alpha \pi}{2}\right)}\left[{ }_{x_{L}} D_{x}^{\alpha} u(x, y, t)+{ }_{x} D_{x_{R}}^{\alpha} u(x, y, t)\right]
$$

in which

$$
{ }_{x_{L}} D_{x}^{\alpha} u(x, y, t)=\frac{1}{\Gamma(2-\alpha)} \frac{\partial^{2}}{\partial x^{2}} \int_{x_{L}}^{x} \frac{u(\eta, y, t)}{(x-\eta)^{\alpha-1}} d \eta, \quad{ }_{x} D_{x_{R}}^{\alpha} u(x, y, t)=\frac{1}{\Gamma(2-\alpha)} \frac{\partial^{2}}{\partial x^{2}} \int_{x}^{x_{R}} \frac{u(\eta, y, t)}{(\eta-x)^{\alpha-1}} d \eta,
$$

are the left-sided and right-sided Riemann-Liouville fractional derivatives in $x$ direction, and $\beta$-order Riesz fractional derivative $\frac{\partial^{\beta} u(x, y, t)}{\partial|y|^{\beta}}$ can be defined similarly. Moreover, it is remarkable that the absorbing boundary condition is imposed on (1.1) based on some actual physical meanings (cf. [1]).

Problems in the form (1.1) cover a series of interesting practical models such as the time fractional advection-dispersion equations (cf. [2]), fractional mobile-immobile advection-dispersion equations (cf. [3$5]$ ), fractional kinetic equations (cf. [6-8]) and fractional Fokker-Planck equations (cf. [9, 10]). To construct a numerical method for solving problem (1.1), the discretizations of the Caputo derivatives and RiemannLiouville derivatives are the key points. For the discretization of Caputo derivatives, a popular method is to use the piecewise linear approximation, i.e. the so-called $L 1$ method (cf. [11-14]). For the discretization of Riemann-Liouville derivatives, Meerschaert \& Tadjeran [15] proposed the shifted Grünwald-Letnikov formula, whose extended/improved versions can be also found in references [16-20]. Nevertheless, since the fractional differential operators are nonlocal, most of the numerical methods for fractional differential equations usually generate dense coefficient matrices, which lead to an expensive computational cost. In order to improve the computational efficiency of the methods, some effective techniques have been developed. For example, Wang, Wang \& Sircar [21] introduced the fast Fourier transform for the methods with Toeplitzlike coefficient matrix. In addition, some other accelerating methods have been also presented, such as the multigrid method, the fast ADI method, the preconditioned conjugate gradient method, the multilevel circulant preconditioned method and the Kronecker product splitting (KPS) method (cf. [22-28]).

Motivated by the above research, for problem (1.1), we here develop an implicit difference scheme along with an accelerating strategy. The paper is organized as follows. In Section 2, an implicit difference scheme for problem (1.1) is proposed. In Section 3, the stability and convergence analysis of the difference scheme are analyzed and thus the corresponding criteria are derived. In Section 4, by combining the KPS preconditioner (cf. [27, 28]) with the GMRES method (cf. [29]), an accelerating strategy is given. Numerical experiments are presented in Section 5 to support the theoretical findings.

\section{An implicit difference scheme}

Let $N_{1}, N_{2}, M \in \mathbb{N}, \tau=\frac{T-t_{0}}{M}, h_{1}=\frac{x_{R}-x_{L}}{N_{1}+1}$ and $h_{2}=\frac{y_{R}-y_{L}}{N_{2}+1}$, and define the following grid sets:

$$
\begin{gathered}
\bar{\Omega}_{h}=\left\{\left(x_{i}, y_{j}\right): x_{i}=x_{L}+i h_{1}, 0 \leq i \leq N_{1}+1 ; y_{j}=y_{L}+j h_{2}, 0 \leq j \leq N_{2}+1\right\}, \\
\Omega_{\tau}=\left\{t_{m}: t_{m}=t_{0}+m \tau, 0 \leq m \leq M\right\}, \Omega_{h}=\bar{\Omega}_{h} \cap \Omega, \partial \Omega_{h}=\bar{\Omega}_{h} \cap \partial \Omega, \Omega_{h \tau}=\Omega_{h} \times \Omega_{\tau} .
\end{gathered}
$$

Under condition: $u(\cdot, \cdot, t) \in C^{(2)}\left(\left[t_{0}, T\right]\right)$, the following equalities hold:

$$
\begin{aligned}
& { }_{t_{0}}^{C} D_{t}^{\gamma} u\left(x, y, t_{m}\right)=\frac{1}{\Gamma(1-\gamma)} \sum_{s=1}^{m} \int_{t_{s-1}}^{t_{s}} \frac{u\left(x, y, t_{s}\right)-u\left(x, y, t_{s-1}\right)}{\tau} \frac{d \xi}{\left(t_{m}-\xi\right)^{\gamma}}+\mathcal{O}\left(\tau^{2-\gamma}\right) \\
= & \frac{\tau^{-\gamma}}{\Gamma(2-\gamma)} \sum_{s=1}^{m} b_{m-s}^{(\gamma)}\left[u\left(x, y, t_{s}\right)-u\left(x, y, t_{s-1}\right)\right]+\mathcal{O}\left(\tau^{2-\gamma}\right),
\end{aligned}
$$

where $b_{s}^{(\gamma)}=(s+1)^{1-\gamma}-s^{1-\gamma}$ satisfies the properties for $s \geq 1$ :

$$
b_{0}^{(\gamma)}=1, \quad b_{s-1}^{(\gamma)}>b_{s}^{(\gamma)}, \quad(1-\gamma) s^{-\gamma}<b_{s-1}^{(\gamma)}<(1-\gamma)(s-1)^{-\gamma} .
$$


When dropping the remainder $\mathcal{O}\left(\tau^{2-\gamma}\right)$ in $(2.1)$, the derived approximation formula can be used for the time discretization of problem (1.1), that is the so-called $L 1$ method (see e.g. [11-14]). Concerning the space discretization of problem (1.1), we may apply (1.3) to compute the Riesz fractional derivatives, in which the Riemann-Liouville fractional derivatives can be approximated by the weighed-shifted Grünwald-Letnikov difference (WSGD) operator (see e.g. [17]).

In view of the absorbing boundary condition in (1.1), we define a function for $y \in\left[y_{L}, y_{R}\right]$ and $t \in\left[t_{0}, T\right]$ :

$$
\hat{u}(x)= \begin{cases}u(x, y, t), & x \in\left[x_{L}, x_{R}\right], \\ 0, & \text { otherwise },\end{cases}
$$

and introduce a set:

$$
\mathcal{L}^{n+\alpha}(\mathbb{R})=\left\{v \in L_{1}(\mathbb{R}): \int_{-\infty}^{+\infty}(1+|k|)^{n+\alpha}\left|\int_{-\infty}^{+\infty} e^{i k x} v(x) d x\right| d k<+\infty\right\} .
$$

Moreover, we also need the following result from Hao, Sun \& Cao [17].

Lemma 2.1. (cf. [17]) Suppose $\hat{u}(x) \in \mathcal{L}^{2+\alpha}(\mathbb{R})$ and set

$$
\begin{gathered}
\lambda_{1}=\frac{\alpha^{2}+3 \alpha+2}{12}, \quad \lambda_{0}=\frac{4-\alpha^{2}}{6}, \quad \lambda_{-1}=\frac{\alpha^{2}-3 \alpha+2}{12}, \quad g_{k}^{(\alpha)}=(-1)^{k}\left(\begin{array}{l}
\alpha \\
k
\end{array}\right), \\
w_{0}^{(\alpha)}=\lambda_{1} g_{0}^{(\alpha)}, \quad w_{1}^{(\alpha)}=\lambda_{1} g_{1}^{(\alpha)}+\lambda_{0} g_{0}^{(\alpha)}, \quad w_{k}^{(\alpha)}=\lambda_{1} g_{k}^{(\alpha)}+\lambda_{0} g_{k-1}^{(\alpha)}+\lambda_{-1} g_{k-2}^{(\alpha)}(k \geq 2), \\
{ }_{L} \delta_{x}^{\alpha} \hat{u}(x)=\frac{1}{h_{1}^{\alpha}} \sum_{k=0}^{\left\lfloor\frac{x-x_{L}}{h_{1}}\right\rfloor} w_{k}^{(\alpha)} \hat{u}\left(x-(k-1) h_{1}\right), \quad{ }_{R} \delta_{x}^{\alpha} \hat{u}(x)=\frac{1}{h_{1}^{\alpha}} \sum_{k=0}^{\left\lfloor\frac{x_{R}-x}{h_{1}}\right\rfloor} w_{k}^{(\alpha)} \hat{u}\left(x+(k-1) h_{1}\right) .
\end{gathered}
$$

Then

$$
{ }_{x_{L}} D_{x}^{\alpha} \hat{u}(x)={ }_{L} \delta_{x}^{\alpha} \hat{u}(x)+\mathcal{O}\left(h_{1}^{2}\right), \quad{ }_{x} D_{x_{R}}^{\alpha} \hat{u}(x)={ }_{R} \delta_{x}^{\alpha} \hat{u}(x)+\mathcal{O}\left(h_{1}^{2}\right) .
$$

Clearly, a result similar to Lemma 2.1 also holds for the variable $y$. Write $U_{i, j}^{m}=u\left(x_{i}, y_{j}, t_{m}\right)$ and $f_{i, j}^{m}=f\left(x_{i}, y_{j}, t_{m}\right)$. Applying (2.1), (2.3) and the Taylor expansion to (1.1) yields

$$
\begin{aligned}
& a_{1} \frac{U_{i, j}^{m}-U_{i, j}^{m-1}}{\tau}+\frac{a_{2} \tau^{-\gamma}}{\Gamma(2-\gamma)} \sum_{s=1}^{m} b_{m-s}^{(\gamma)}\left(U_{i, j}^{s}-U_{i, j}^{s-1}\right) \\
= & a_{3} \frac{U_{i+1, j}^{m}-U_{i-1, j}^{m}}{2 h_{1}}+a_{4} \frac{U_{i, j+1}^{m}-U_{i, j-1}^{m}}{2 h_{2}}-\frac{a_{5}}{2 \cos \left(\frac{\alpha \pi}{2}\right) h_{1}^{\alpha}}\left[\sum_{k=0}^{i} w_{k}^{(\alpha)} U_{i-k+1, j}^{m}+\sum_{k=0}^{N_{1}-i+1} w_{k}^{(\alpha)} U_{i+k-1, j}^{m}\right] \\
& -\frac{a_{6}}{2 \cos \left(\frac{\beta \pi}{2}\right) h_{2}^{\beta}}\left[\sum_{l=0}^{j} w_{l}^{(\beta)} U_{i, j-l+1}^{m}+\sum_{l=0}^{N_{2}-j+1} w_{l}^{(\beta)} U_{i, j+l-1}^{m}\right]+f_{i, j}^{m}+R_{i, j}^{m}, \quad\left(x_{i}, y_{j}, t_{m}\right) \in \Omega_{h \tau},
\end{aligned}
$$

where

$$
\left|R_{i, j}^{m}\right|= \begin{cases}\mathcal{O}\left(\tau+h_{1}^{2}+h_{2}^{2}\right), & \text { when } a_{1} \neq 0, \\ \mathcal{O}\left(\tau^{2-\gamma}+h_{1}^{2}+h_{2}^{2}\right), & \text { when } a_{1}=0 \text { and } a_{2} \neq 0 .\end{cases}
$$

Omitting the remainder $R_{i, j}^{m}$ in (2.4) and replacing $U_{i, j}^{m}$ by the corresponding numerical approximation $u_{i, j}^{m}$, an implicit difference scheme for (1.1) can be derived as follows:

$$
a_{1} \frac{u_{i, j}^{m}-u_{i, j}^{m-1}}{\tau}+\frac{a_{2} \tau^{-\gamma}}{\Gamma(2-\gamma)} \sum_{s=1}^{m} b_{m-s}^{(\gamma)}\left(u_{i, j}^{s}-u_{i, j}^{s-1}\right)
$$




$$
\begin{aligned}
= & a_{3} \frac{u_{i+1, j}^{m}-u_{i-1, j}^{m}}{2 h_{1}}+a_{4} \frac{u_{i, j+1}^{m}-u_{i, j-1}^{m}}{2 h_{2}}-\frac{a_{5}}{2 \cos \left(\frac{\alpha \pi}{2}\right) h_{1}^{\alpha}}\left[\sum_{k=0}^{i} w_{k}^{(\alpha)} u_{i-k+1, j}^{m}+\sum_{k=0}^{N_{1}-i+1} w_{k}^{(\alpha)} u_{i+k-1, j}^{m}\right] \\
& -\frac{a_{6}}{2 \cos \left(\frac{\beta \pi}{2}\right) h_{2}^{\beta}}\left[\sum_{l=0}^{j} w_{l}^{(\beta)} u_{i, j-l+1}^{m}+\sum_{l=0}^{N_{2}-j+1} w_{l}^{(\beta)} u_{i, j+l-1}^{m}\right]+f_{i, j}^{m}, \quad\left(x_{i}, y_{j}, t_{m}\right) \in \Omega_{h \tau},
\end{aligned}
$$

where the initial and boundary values are respectively given by

$$
u_{i, j}^{0}=u_{0}\left(x_{i}, y_{j}\right),\left(x_{i}, y_{j}\right) \in \Omega_{h} ; \quad u_{i, j}^{m}=0,\left(x_{i}, y_{j}\right) \in \partial \Omega_{h}, 0 \leq m \leq M .
$$

\section{Stability and convergence of the implicit difference scheme}

This section will deal with the stability and convergence of the implicit difference scheme (2.6)-(2.7). Let

$$
V_{h}=\left\{v: v=\left\{v_{i, j}\right\} \text { is a grid function on } \bar{\Omega}_{h} \text { and } v_{i, j}=0 \text { if }\left(x_{i}, y_{j}\right) \in \partial \Omega_{h}\right\} .
$$

On $V_{h}$ we define the following inner product and the corresponding norm:

$$
(u, v)=h_{1} h_{2} \sum_{i=1}^{N_{1}} \sum_{j=1}^{N_{2}} u_{i, j} v_{i, j}, \quad\|u\|=\sqrt{(u, u)}, \quad \forall u, v \in V_{h} .
$$

The following lemma from Vong, Lyu, Chen \& Lei [30] will play an important role in our analysis.

Lemma 3.1. (cf. [30]) Let $\alpha \in(1,2), N_{1} \geq 5$ and $v \in V_{h}$. Then

$$
\left({ }_{L} \delta_{x}^{\alpha} v, v\right)+\left({ }_{R} \delta_{x}^{\alpha} v, v\right)=h_{1}^{1-\alpha} h_{2} \sum_{i=1}^{N_{1}} \sum_{j=1}^{N_{2}}\left[\sum_{k=0}^{i} w_{k}^{(\alpha)} v_{i-k+1, j}+\sum_{k=0}^{N_{1}-i+1} w_{k}^{(\alpha)} v_{i+k-1, j}\right] v_{i, j} \leq-\ln 2 c_{1}\|v\|^{2},
$$

where $c_{1}>0$ is a constant independent of the stepsizes $h_{1}$ and $h_{2}$.

Introducing the following notations for $0 \leq m \leq M$ :

$$
\begin{gathered}
u^{m}=\left(u_{1,1}^{m}, u_{2,1}^{m}, \ldots, u_{N_{1}, 1}^{m}, u_{1,2}^{m}, u_{2,2}^{m}, \ldots, u_{N_{1}, 2}^{m}, \ldots, u_{1, N_{2}}^{m}, u_{2, N_{2}}^{m}, \ldots, u_{N_{1}, N_{2}}^{m}\right)^{T}, \\
f^{m}=\left(f_{1,1}^{m}, f_{2,1}^{m}, \ldots, f_{N_{1}, 1}^{m}, f_{1,2}^{m}, f_{2,2}^{m}, \ldots, f_{N_{1}, 2}^{m}, \ldots, f_{1, N_{2}}^{m}, f_{2, N_{2}}^{m}, \ldots, f_{N_{1}, N_{2}}^{m}\right)^{T},
\end{gathered}
$$

a stability criterion can be derived under the condition: $\left|a_{1}\right|+\left|a_{2}\right| \neq 0$.

Theorem 3.2. Let $N_{1}, N_{2} \geq 5$. Then implicit difference scheme (2.6)-(2.7) is stable with respect to the initial value $u^{0}$ and source term $f$, namely, the following stability inequalities hold for $1 \leq m \leq M$ :

$$
\begin{aligned}
& \left\|u^{m}\right\|^{2} \leq \begin{cases}\left\|u^{0}\right\|^{2}+\frac{\tau}{2 \ln 2 a_{1} c_{2}} \sum_{k=1}^{m}\left\|f^{k}\right\|^{2}, & \text { when } a_{1} \neq 0 \text { and } a_{2}=0, \\
\left\|u^{0}\right\|^{2}+\frac{\left(T-t_{0}\right)^{\gamma} \Gamma(1-\gamma)}{2 \ln 2 a_{2} c_{2}} \max _{1 \leq k \leq m}\left\|f^{k}\right\|^{2}, & \text { when } a_{1} \neq 0 \text { and } a_{2} \neq 0\end{cases} \\
& \left\|u^{m}\right\|^{2} \leq\left\|u^{0}\right\|^{2}+\frac{\left(T-t_{0}\right)^{\gamma} \Gamma(1-\gamma)}{2 \ln 2 a_{2} c_{2}} \max _{1 \leq k \leq m}\left\|f^{k}\right\|^{2} \text {, when } a_{1}=0 \text { and } a_{2} \neq 0,
\end{aligned}
$$

where $c_{2}>0$ is a constant independent of the stepsizes $h_{1}, h_{2}$ and $\tau$. 
Proof. Multiplying the both sides of (2.6) by $h_{1} h_{2} u_{i, j}^{m}$ and then summing for $i=1,2, \ldots, N_{1}$ and $j=1,2, \ldots, N_{2}$ yield

$$
\begin{aligned}
& {\left[a_{1}+\frac{a_{2} \tau^{1-\gamma}}{\Gamma(2-\gamma)}\right] h_{1} h_{2} \sum_{i=1}^{N_{1}} \sum_{j=1}^{N_{2}}\left(u_{i, j}^{m}\right)^{2}-\frac{a_{3} \tau h_{2}}{2} \sum_{i=1}^{N_{1}} \sum_{j=1}^{N_{2}}\left(u_{i+1, j}^{m}-u_{i-1, j}^{m}\right) u_{i, j}^{m}-\frac{a_{4} \tau h_{1}}{2} \sum_{i=1}^{N_{1}} \sum_{j=1}^{N_{2}}\left(u_{i, j+1}^{m}-u_{i, j-1}^{m}\right) u_{i, j}^{m} } \\
= & a_{1} h_{1} h_{2} \sum_{i=1}^{N_{1}} \sum_{j=1}^{N_{2}} u_{i, j}^{m-1} u_{i, j}^{m}+\frac{a_{2} \tau^{1-\gamma} h_{1} h_{2}}{\Gamma(2-\gamma)} \sum_{i=1}^{N_{1}} \sum_{j=1}^{N_{2}}\left[b_{m-1}^{(\gamma)} u_{i, j}^{0}+\sum_{s=1}^{m-1}\left(b_{m-s-1}^{(\gamma)}-b_{m-s}^{(\gamma)}\right) u_{i, j}^{s}\right] u_{i, j}^{m} \\
& -\frac{a_{5} \tau h_{1}^{1-\alpha} h_{2}}{2 \cos \left(\frac{\alpha \pi}{2}\right)} \sum_{i=1}^{N_{1}} \sum_{j=1}^{N_{2}}\left(\sum_{k=0}^{i} w_{k}^{(\alpha)} u_{i-k+1, j}^{m}+\sum_{k=0}^{N_{1}-i+1} w_{k}^{(\alpha)} u_{i+k-1, j}^{m}\right) u_{i, j}^{m} \\
& -\frac{a_{6} \tau h_{1} h_{2}^{1-\beta}}{2 \cos \left(\frac{\beta \pi}{2}\right)} \sum_{i=1}^{N_{1}} \sum_{j=1}^{N_{2}}\left(\sum_{l=0}^{j} w_{l}^{(\beta)} u_{i, j-l+1}^{m}+\sum_{l=0}^{N_{2}-j+1} w_{l}^{(\beta)} u_{i, j+l-1}^{m}\right) u_{i, j}^{m}+\tau h_{1} h_{2} \sum_{i=1}^{N_{1}} \sum_{j=1}^{N_{2}} f_{i, j}^{m} u_{i, j}^{m} .
\end{aligned}
$$

Since $u_{0, j}^{m}=u_{N_{1}+1, j}^{m}=u_{i, 0}^{m}=u_{i, N_{2}+1}^{m}=0$ for $0 \leq i \leq N_{1}+1,0 \leq j \leq N_{2}+1$ and $0 \leq m \leq M$, we have that

$$
\sum_{i=1}^{N_{1}} \sum_{j=1}^{N_{2}}\left(u_{i+1, j}^{m}-u_{i-1, j}^{m}\right) u_{i, j}^{m}=0, \quad \sum_{i=1}^{N_{1}} \sum_{j=1}^{N_{2}}\left(u_{i, j+1}^{m}-u_{i, j-1}^{m}\right) u_{i, j}^{m}=0 .
$$

Applying (2.2), (3.4), Lemma 3.1 and the Cauchy-Schwartz inequality to (3.3) implies that, for all $\epsilon>0$,

$$
\begin{aligned}
{\left[\frac{a_{1}}{2}+\frac{a_{2} \tau^{1-\gamma}}{\Gamma(2-\gamma)}\right]\left\|u^{m}\right\|^{2} \leq } & \frac{a_{1}}{2}\left\|u^{m-1}\right\|^{2}+\frac{a_{2} \tau^{1-\gamma}}{2 \Gamma(2-\gamma)}\left[b_{m-1}^{(\gamma)}\left\|u^{0}\right\|^{2}+\sum_{s=1}^{m-1}\left(b_{m-s-1}^{(\gamma)}-b_{m-s}^{(\gamma)}\right)\left\|u^{s}\right\|^{2}+\left\|u^{m}\right\|^{2}\right] \\
& -\ln 2 \tau c_{2}\left\|u^{m}\right\|^{2}+\tau\left(\epsilon\left\|u^{m}\right\|^{2}+\frac{1}{4 \epsilon}\left\|f^{m}\right\|^{2}\right)
\end{aligned}
$$

where $c_{2}=-\frac{a_{5}}{2 \cos \left(\frac{\alpha \pi}{2}\right)}-\frac{a_{6}}{2 \cos \left(\frac{\beta \pi}{2}\right)}$. Letting $\epsilon=\ln 2 c_{2}$ in (3.5), we obtain

$$
\left[a_{1}+\frac{a_{2} \tau^{1-\gamma}}{\Gamma(2-\gamma)}\right]\left\|u^{m}\right\|^{2} \leq a_{1}\left\|u^{m-1}\right\|^{2}+\frac{a_{2} \tau^{1-\gamma}}{\Gamma(2-\gamma)}\left[\sum_{s=1}^{m-1}\left(b_{m-s-1}^{(\gamma)}-b_{m-s}^{(\gamma)}\right)\left\|u^{s}\right\|^{2}+b_{m-1}^{(\gamma)}\left\|u^{0}\right\|^{2}\right]+\frac{\tau\left\|f^{m}\right\|^{2}}{2 \ln 2 c_{2}} .
$$

Setting $a_{2}=0$ in (3.6), the first inequality of (3.1) follows immediately by recursion. Next, we prove the second inequality of (3.1) by mathematical induction. When $a_{1}, a_{2} \neq 0$, from (2.2) and (3.6) one obtains

$$
\begin{aligned}
{\left[a_{1}+\frac{a_{2} \tau^{1-\gamma}}{\Gamma(2-\gamma)}\right]\left\|u^{m}\right\|^{2} \leq } & a_{1}\left\|u^{m-1}\right\|^{2}+\frac{a_{2} \tau^{1-\gamma}}{\Gamma(2-\gamma)} \sum_{s=1}^{m-1}\left(b_{m-s-1}^{(\gamma)}-b_{m-s}^{(\gamma)}\right)\left\|u^{s}\right\|^{2} \\
& +\frac{a_{2} \tau^{1-\gamma}}{\Gamma(2-\gamma)} b_{m-1}^{(\gamma)}\left[\left\|u^{0}\right\|^{2}+\frac{\left(T-t_{0}\right)^{\gamma} \Gamma(1-\gamma)}{2 \ln 2 a_{2} c_{2}}\left\|f^{m}\right\|^{2}\right] .
\end{aligned}
$$

Write $P_{m}=\left\|u^{0}\right\|^{2}+\frac{\left(T-t_{0}\right)^{\gamma} \Gamma(1-\gamma)}{2 \ln 2 a_{2} c_{2}} \max _{1 \leq k \leq m}\left\|f^{k}\right\|^{2}$. Then from (3.7) it follows that

$$
\left\|u^{1}\right\|^{2} \leq\left\|u^{0}\right\|^{2}+\frac{\Gamma(2-\gamma) \tau}{\left[a_{1} \Gamma(2-\gamma)+a_{2} \tau^{1-\gamma}\right] 2 \ln 2 c_{2}}\left\|f^{1}\right\|^{2} \leq\left\|u^{0}\right\|^{2}+\frac{\tau^{\gamma} \Gamma(2-\gamma)}{2 \ln 2 a_{2} c_{2}}\left\|f^{1}\right\|^{2} \leq P_{1} .
$$

Assume now that the second inequality of $(3.1)$ holds for $m=2,3, \ldots, n(1 \leq n<M)$. Then, when $m=n+1$, from (3.7) and the induction hypothesis, one has

$$
\left[a_{1}+\frac{a_{2} \tau^{1-\gamma}}{\Gamma(2-\gamma)}\right]\left\|u^{n+1}\right\|^{2} \leq a_{1} P_{n}+\frac{a_{2} \tau^{1-\gamma}}{\Gamma(2-\gamma)}\left[\sum_{s=1}^{n}\left(b_{n-s}^{(\gamma)}-b_{n-s+1}^{(\gamma)}\right) P_{s}+b_{n}^{(\gamma)} P_{n+1}\right]
$$




$$
\leq a_{1} P_{n+1}+\frac{a_{2} \tau^{1-\gamma}}{\Gamma(2-\gamma)}\left[\sum_{s=1}^{n}\left(b_{n-s}^{(\gamma)}-b_{n-s+1}^{(\gamma)}\right) P_{n+1}+b_{n}^{(\gamma)} P_{n+1}\right]=\left[a_{1}+\frac{a_{2} \tau^{1-\gamma}}{\Gamma(2-\gamma)}\right] P_{n+1},
$$

which implies $\left\|u^{n+1}\right\|^{2} \leq P_{n+1}$. Hence the second inequality of (3.1) is proved.

When $a_{1}=0$ and $a_{2} \neq 0$, from (2.2) and (3.6) one deduces:

$$
\left\|u^{m}\right\|^{2} \leq \sum_{s=1}^{m-1}\left(b_{m-s-1}^{(\gamma)}-b_{m-s}^{(\gamma)}\right)\left\|u^{s}\right\|^{2}+b_{m-1}^{(\gamma)}\left[\left\|u^{0}\right\|^{2}+\frac{\left(T-t_{0}\right)^{\gamma} \Gamma(1-\gamma)}{2 \ln 2 a_{2} c_{2}}\left\|f^{m}\right\|^{2}\right] .
$$

Based on (3.8) and using a similar proof for the second inequality of (3.1), inequality (3.2) can be also derived. This completes the proof.

Let

$$
\begin{gathered}
e_{i, j}^{m}=U_{i, j}^{m}-u_{i, j}^{m}, \quad e^{m}=\left(e_{1,1}^{m}, e_{2,1}^{m}, \ldots, e_{N_{1}, 1}^{m}, e_{1,2}^{m}, e_{2,2}^{m}, \ldots, e_{N_{1}, 2}^{m}, \ldots, e_{1, N_{2}}^{m}, e_{2, N_{2}}^{m}, \ldots, e_{N_{1}, N_{2}}^{m}\right)^{T}, \\
R^{m}=\left(R_{1,1}^{m}, R_{2,1}^{m}, \ldots, R_{N_{1}, 1}^{m}, R_{1,2}^{m}, R_{2,2}^{m}, \ldots, R_{N_{1}, 2}^{m}, \ldots, R_{1, N_{2}}^{m}, R_{2, N_{2}}^{m}, \ldots, R_{N_{1}, N_{2}}^{m}\right)^{T},
\end{gathered}
$$

Then, under the condition: $\left|a_{1}\right|+\left|a_{2}\right| \neq 0$, scheme (2.6)-(2.7) can be proved to be convergent.

Theorem 3.3. The implicit difference scheme (2.6)-(2.7) has the following error estimates for $1 \leq m \leq M$ :

$$
\left\|e^{m}\right\|= \begin{cases}\mathcal{O}\left(\tau+h_{1}^{2}+h_{2}^{2}\right), & \text { when } a_{1} \neq 0, \\ \mathcal{O}\left(\tau^{2-\gamma}+h_{1}^{2}+h_{2}^{2}\right), & \text { when } a_{1}=0 \text { and } a_{2} \neq 0 .\end{cases}
$$

Proof. Subtracting (2.6) from (2.4) yields

$$
\begin{aligned}
& a_{1} \frac{e_{i, j}^{m}-e_{i, j}^{m-1}}{\tau}+\frac{a_{2} \tau^{-\gamma}}{\Gamma(2-\gamma)} \sum_{s=1}^{m} b_{m-s}^{(\gamma)}\left(e_{i, j}^{s}-e_{i, j}^{s-1}\right) \\
= & a_{3} \frac{e_{i+1, j}^{m}-e_{i-1, j}^{m}}{2 h_{1}}+a_{4} \frac{e_{i, j+1}^{m}-e_{i, j-1}^{m}}{2 h_{2}}-\frac{a_{5}}{2 \cos \left(\frac{\alpha \pi}{2}\right) h_{1}^{\alpha}}\left[\sum_{k=0}^{i} w_{k}^{(\alpha)} e_{i-k+1, j}^{m}+\sum_{k=0}^{N_{1}-i+1} w_{k}^{(\alpha)} e_{i+k-1, j}^{m}\right] \\
& -\frac{a_{6}}{2 \cos \left(\frac{\beta \pi}{2}\right) h_{2}^{\beta}}\left[\sum_{l=0}^{j} w_{l}^{(\beta)} e_{i, j-l+1}^{m}+\sum_{l=0}^{N_{2}-j+1} w_{l}^{(\beta)} e_{i, j+l-1}^{m}\right]+R_{i, j}^{m}, \quad\left(x_{i}, y_{j}, t_{m}\right) \in \Omega_{h \tau} .
\end{aligned}
$$

Since by (2.5) there exists $c_{0}>0$ such that, for all $i, j, m$,

$$
\left|R_{i, j}^{m}\right| \leq \begin{cases}c_{0}\left(\tau+h_{1}^{2}+h_{2}^{2}\right), & \text { when } a_{1} \neq 0, \\ c_{0}\left(\tau^{2-\gamma}+h_{1}^{2}+h_{2}^{2}\right), & \text { when } a_{1}=0 \text { and } a_{2} \neq 0,\end{cases}
$$

a similar derivation process as in Theorem 3.2 shows that:

- when $a_{1} \neq 0$ and $a_{2}=0$,

$$
\left\|e^{m}\right\| \leq \sqrt{\frac{\tau}{2 \ln 2 a_{1} c_{2}} \sum_{k=1}^{m}\left\|R^{k}\right\|^{2}} \leq \sqrt{\frac{c_{0}^{2}\left(T-t_{0}\right)\left(x_{R}-x_{L}\right)\left(y_{R}-y_{L}\right)}{2 \ln 2 a_{1} c_{2}}}\left(\tau+h_{1}^{2}+h_{2}^{2}\right) ;
$$

- when $a_{1} \neq 0$ and $a_{2} \neq 0$,

$$
\left\|e^{m}\right\| \leq \sqrt{\frac{\left(T-t_{0}\right)^{\gamma} \Gamma(1-\gamma)}{2 \ln 2 a_{2} c_{2}}} \max _{1 \leq k \leq m}\left\|R^{k}\right\| \leq \sqrt{\frac{c_{0}^{2}\left(T-t_{0}\right)^{\gamma}\left(x_{R}-x_{L}\right)\left(y_{R}-y_{L}\right) \Gamma(1-\gamma)}{2 \ln 2 a_{2} c_{2}}}\left(\tau+h_{1}^{2}+h_{2}^{2}\right) ;
$$


- when $a_{1}=0$ and $a_{2} \neq 0$,

$$
\left\|e^{m}\right\| \leq \sqrt{\frac{\left(T-t_{0}\right)^{\gamma} \Gamma(1-\gamma)}{2 \ln 2 a_{2} c_{2}}} \max _{1 \leq k \leq m}\left\|R^{k}\right\| \leq \sqrt{\frac{c_{0}^{2}\left(T-t_{0}\right)^{\gamma}\left(x_{R}-x_{L}\right)\left(y_{R}-y_{L}\right) \Gamma(1-\gamma)}{2 \ln 2 a_{2} c_{2}}}\left(\tau^{2-\gamma}+h_{1}^{2}+h_{2}^{2}\right) .
$$

Therefore, the theorem is proved.

\section{GMRES method with the KPS preconditioner}

Generally speaking, when the scheme (2.6)-(2.7) is applied to problem (1.1), a large-scale linear system will emerge, which needs an expensive computational cost. In order to improve the computational efficiency, in this section, we will consider an efficient implementation strategy by using the GMRES method (cf. [29]) with the KPS preconditioner (cf. [27, 28]).

Let $I_{N_{i}}$ be the $N_{i} \times N_{i}$ identity matrix, $B_{N_{i}}=\operatorname{tridiag}\{-1,0,1\} \in \mathbb{R}^{N_{i} \times N_{i}}$, and set

$$
\begin{aligned}
& d_{1}=\frac{a_{2} \tau^{1-\gamma}}{\Gamma(2-\gamma)}, \quad d_{2}=-\frac{a_{3} \tau}{2 h_{1}}, \quad d_{3}=-\frac{a_{4} \tau}{2 h_{2}}, \quad d_{4}=\frac{a_{5} \tau}{2 \cos \left(\frac{\alpha \pi}{2}\right) h_{1}^{\alpha}}, \quad d_{5}=\frac{a_{6} \tau}{2 \cos \left(\frac{\beta \pi}{2}\right) h_{2}^{\beta}}, \\
& \mathcal{U}_{m}=u^{m}, \quad \mathcal{G}_{m}=a_{1} u^{m-1}+d_{1}\left[b_{m-1}^{(\gamma)} u^{0}+\sum_{s=1}^{m-1}\left(b_{m-s-1}^{(\gamma)}-b_{m-s}^{(\gamma)}\right) u^{s}\right]+\tau f^{m}, \\
& W_{\xi}=\left[\begin{array}{ccccc}
w_{1}^{(\xi)} & w_{0}^{(\xi)} & & & \\
w_{2}^{(\xi)} & w_{1}^{(\xi)} & w_{0}^{(\xi)} & & \\
\vdots & \vdots & \ddots & \ddots & \\
w_{N_{i}-1}^{(\xi)} & w_{N_{i}-2}^{(\xi)} & \cdots & w_{1}^{(\xi)} & w_{0}^{(\xi)} \\
w_{N_{i}}^{(\xi)} & w_{N_{i}-1}^{(\xi)} & \cdots & w_{2}^{(\xi)} & w_{1}^{(\xi)}
\end{array}\right], \text { where }(\xi, i)=(\alpha, 1) \operatorname{or}(\beta, 2)
\end{aligned}
$$

With the above notations, scheme (2.6) can be cast into the following form with $m=1,2, \ldots, M$ :

$$
\left[\left(a_{1}+d_{1}\right) I_{N_{1} N_{2}}+I_{N_{2}} \otimes\left(d_{2} B_{N_{1}}+d_{4} W_{\alpha}+d_{4} W_{\alpha}^{T}\right)+\left(d_{3} B_{N_{2}}+d_{5} W_{\beta}+d_{5} W_{\beta}^{T}\right) \otimes I_{N_{1}}\right] \mathcal{U}_{m}=\mathcal{G}_{m} .
$$

Scheme (4.1) is an $N_{1} N_{2}$-dimensional linear system, which may be very large when $N_{1}$ and/or $N_{2}$ is large. Thus, applying a classical direct method to solve (4.1), a heavy computational cost would be required. In order to accelerate the computation of scheme (4.1), in the following, we will give a preconditioned method. Write

$$
K_{\alpha}=\left(\frac{a_{1}+d_{1}}{2}\right) I_{N_{1}}+d_{2} B_{N_{1}}+d_{4} W_{\alpha}+d_{4} W_{\alpha}^{T}, \quad K_{\beta}=\left(\frac{a_{1}+d_{1}}{2}\right) I_{N_{2}}+d_{3} B_{N_{2}}+d_{5} W_{\beta}+d_{5} W_{\beta}^{T} .
$$

Then, the coefficient matrix in (4.1) reads $A:=I_{N_{2}} \otimes K_{\alpha}+K_{\beta} \otimes I_{N_{1}}$, and can be split into the following form with parameters $\theta_{1}, \theta_{2}>0$ :

$$
A=P\left(\theta_{1}, \theta_{2}\right)-R\left(\theta_{1}, \theta_{2}\right)
$$

where

$$
P\left(\theta_{1}, \theta_{2}\right)=\frac{1}{\theta_{1}+\theta_{2}}\left(\theta_{1} I_{N_{2}}+K_{\beta}\right) \otimes\left(\theta_{2} I_{N_{1}}+K_{\alpha}\right), \quad R\left(\theta_{1}, \theta_{2}\right)=\frac{1}{\theta_{1}+\theta_{2}}\left(\theta_{2} I_{N_{2}}-K_{\beta}\right) \otimes\left(\theta_{1} I_{N_{1}}-K_{\alpha}\right) .
$$

In our preconditioned method, $P\left(\theta_{1}, \theta_{2}\right)$ will be considered as a preconditioner: its invertibility is assured by the following criterion. 
Lemma 4.1. Assume that $\theta_{1}, \theta_{2}$ are two positive parameters subject to

$$
-\min _{\nu \in \sigma\left(K_{\alpha}\right)} \Re(\nu)<\frac{\theta_{2}-\theta_{1}}{2} \leq \min _{\mu \in \sigma\left(K_{\beta}\right)} \Re(\mu),
$$

where $\sigma(\cdot)$ denotes the spectrum of the given matrix. Then $P\left(\theta_{1}, \theta_{2}\right)$ is invertible.

Proof. By the well-known properties of the Kronecker product (see e.g. [31]), the eigenvalues of $P\left(\theta_{1}, \theta_{2}\right)$ are given:

$$
\lambda=\frac{\left(\theta_{1}+\mu\right)\left(\theta_{2}+\nu\right)}{\theta_{1}+\theta_{2}}=\frac{1}{\theta_{1}+\theta_{2}}\left(\frac{\theta_{1}+\theta_{2}}{2}-\frac{\theta_{2}-\theta_{1}}{2}+\mu\right)\left(\frac{\theta_{1}+\theta_{2}}{2}+\frac{\theta_{2}-\theta_{1}}{2}+\nu\right), \quad \nu \in \sigma\left(K_{\alpha}\right), \mu \in \sigma\left(K_{\beta}\right) .
$$

Then, from (4.3), it follows that $\mu-\frac{\theta_{2}-\theta_{1}}{2} \geq 0$ and $\nu+\frac{\theta_{2}-\theta_{1}}{2}>0$. This, together with $\theta_{1}, \theta_{2}>0$, implies that $\lambda>0$. Hence $P\left(\theta_{1}, \theta_{2}\right)$ is invertible.

When $P\left(\theta_{1}, \theta_{2}\right)$ is invertible, we write $G\left(\theta_{1}, \theta_{2}\right)=P^{-1}\left(\theta_{1}, \theta_{2}\right) R\left(\theta_{1}, \theta_{2}\right)$, that is

$$
G\left(\theta_{1}, \theta_{2}\right)=\left[\left(\theta_{1} I_{N_{2}}+K_{\beta}\right)^{-1}\left(\theta_{2} I_{N_{2}}-K_{\beta}\right)\right] \otimes\left[\left(\theta_{2} I_{N_{1}}+K_{\alpha}\right)^{-1}\left(\theta_{1} I_{N_{1}}-K_{\alpha}\right)\right] .
$$

Then $P^{-1}\left(\theta_{1}, \theta_{2}\right) A=I_{N_{1} N_{2}}-G\left(\theta_{1}, \theta_{2}\right)$ and thus scheme (4.1) is equivalent to

$$
P^{-1}\left(\theta_{1}, \theta_{2}\right) A \mathcal{U}_{m}=\left[I_{N_{1} N_{2}}-G\left(\theta_{1}, \theta_{2}\right)\right] \mathcal{U}_{m}=P^{-1}\left(\theta_{1}, \theta_{2}\right) \mathcal{G}_{m}, \quad m=1,2, \ldots, M .
$$

System (4.4) can be solved by using the GMRES method, where $P\left(\theta_{1}, \theta_{2}\right)$ is a preconditioner (i.e. KPS preconditioner). By using the similar proofs as those of Theorems 3.1 and 3.2 in [27], we can derive the following result.

Theorem 4.2. Assume that $\theta_{1}, \theta_{2}$ are two positive parameters subject to (4.3). Then the spectral radius $\rho\left(G\left(\theta_{1}, \theta_{2}\right)\right)$ of matrix $G\left(\theta_{1}, \theta_{2}\right)$ satisfies that

$$
\rho\left(G\left(\theta_{1}, \theta_{2}\right)\right) \leq r\left(\theta_{1}, \theta_{2}\right):=\max _{\nu \in \sigma\left(K_{\alpha}+\frac{\theta_{2}-\theta_{1}}{2} I_{N_{1}}\right)}\left|\frac{\frac{\theta_{1}+\theta_{2}}{2}-\nu}{\frac{\theta_{1}+\theta_{2}}{2}+\nu}\right|<1,
$$

and the minimum value $r\left(\theta_{1}^{*}, \theta_{2}^{*}\right)$ of the function $r\left(\theta_{1}, \theta_{2}\right)$ is given by

$$
r\left(\theta_{1}^{*}, \theta_{2}^{*}\right)=\left(\sqrt{\frac{\nu_{\max }+\mu_{\min }}{\nu_{\min }+\mu_{\min }}}-1\right)\left(\sqrt{\frac{\nu_{\max }+\mu_{\min }}{\nu_{\min }+\mu_{\min }}}+1\right)^{-1}
$$

where

$$
\theta_{1}^{*}=\sqrt{\left(\nu_{\min }+\mu_{\min }\right)\left(\nu_{\max }+\mu_{\min }\right)}-\mu_{\min }, \quad \theta_{2}^{*}=\sqrt{\left(\nu_{\min }+\mu_{\min }\right)\left(\nu_{\max }+\mu_{\min }\right)}+\mu_{\min },
$$

in which $\nu_{\min }$ and $\nu_{\max }$ denote the minimum and maximum eigenvalues of $K_{\alpha}$, respectively, and $\mu_{\min }$ is the minimum eigenvalue of $K_{\beta}$.

It follows from Theorem 4.2 that, for all positive parameters $\theta_{1}, \theta_{2}$ satisfying (4.3), the eigenvalues of $P^{-1}\left(\theta_{1}, \theta_{2}\right) A$ are located in a circle centered at $(1,0)$ with radius smaller than 1 . This shows that the preconditioner $P\left(\theta_{1}, \theta_{2}\right)$ can accelerate the convergence rate of the GMRES method since a clustered spectrum often translates in rapid convergence of the GMRES method (see e.g. [32]). 


\section{Numerical experiments}

In this section, we present some numerical experiments to illustrate the computational accuracy and efficiency of the GMRES method with the KPS preconditioner for solving the difference scheme (2.6) or (4.1), where the parameters $\theta_{1}$ and $\theta_{2}$ will be taken as the optimal ones indicated in Theorem 4.2. In order to show the computational advantage of the preconditioned method, we will also give a comparison of the following three methods for solving (4.1):

- Method I: using the matrix left-division command in MATLAB;

- Method II: GMRES method without preconditioner;

- Method III: GMRES method with the KPS preconditioner.

The initial guesses for Methods II-III are taken as their approximation at the previous time-step, the restarting value is chosen as 20 and the stopping criterion of the iteration is

$$
\frac{\left\|\mathcal{G}_{m}-A \mathcal{U}_{m}^{(k)}\right\|}{\left\|\mathcal{G}_{m}-A \mathcal{U}_{m}^{(0)}\right\|} \leq 10^{-6}
$$

where $\mathcal{U}_{m}^{(k)}$ denotes the $k$ th-approximation to $\mathcal{U}_{m}$. Moreover, we will always set $h_{1}=h_{2}=h$ and compute the global error and the temporal and spatial convergence orders respectively by the following formulas:

$$
E(h, \tau)=\max _{0 \leq m \leq M}\left\|e^{m}\right\|, \quad \operatorname{Order}_{1}=\log _{2}\left[\frac{E(h, \tau)}{E(h, \tau / 2)}\right], \quad \operatorname{Order}_{2}=\log _{2}\left[\frac{E(h, \tau)}{E(h / 2, \tau)}\right] .
$$

Example 5.1. Consider the initial-boundary problem in the form (1.1) with

$$
a_{1}=a_{2}=a_{5}=a_{6}=1, a_{3}=a_{4}=-1, u_{0}(x, y)=[x(2-x) y(2-y)]^{2}, t_{0}=0, T=1, \Omega=(0,2) \times(0,2),
$$

and source function $f(x, y, t)$ being assigned such that the problems have a common exact solution $u(x, y, t)=$ $\exp (-t)[x(2-x) y(2-y)]^{2}$. For convenience, we write the discrete systems (4.1) corresponding to the above problems as $\mathcal{Q}_{1}(\alpha, \beta, \gamma)$, where $\alpha, \beta, \gamma$ are (suitable) free parameters.

Applying Methods I-III with $h=1 / 60$ and $\tau=1 / 3,1 / 6,1 / 12,1 / 24($ resp. $h=1 / 3,1 / 6,1 / 12,1 / 24$ and $\tau=1 / 1000)$ to the discrete systems $\mathcal{Q}_{1}(1.2,1.8, \gamma)$ with $\gamma=0.1,0.5,0.9$ (resp. $\mathcal{Q}_{1}(\alpha, \beta, 0.5)$ with $(\alpha, \beta)=(1.2,1.8),(1.4,1.6),(1.5,1.5))$, the CPU times (in second), global errors and convergence rates in time (resp. in space) are listed in Table 1 (resp. Table 2). It can be seen from Tables 1-2 that Methods I-III almost have the same accuracy under the same spatial and temporal stepsizes, and can reach the theoretical accuracy stated in Theorem 3.3. By comparing the CPU times, we can find that the computational efficiency of Method III is optimal among the three methods.

The average iteration numbers per time-step versus the different parameters $\theta_{1}, \theta_{2}$ of Method III for $\mathcal{Q}_{1}(1.2,1.8,0.5)$ with $h=\tau=1 / 16$ (resp. $h=\tau=1 / 32$ ) is plotted in Figure 1 (a) (resp. Figure 1 (b)). From Figure 1 , we can observe that there is a good range of parameters $\theta_{1}, \theta_{2}$ (including $\theta_{1}^{*}, \theta_{2}^{*}$ ) for the convergence of Method III. This also implies that the selection of parameters $\theta_{1}^{*}$ and $\theta_{2}^{*}$ in Method III is appropriate. In Figure 2 (a)-(b), we display the spectrums of matrices $A$ and $P^{-1}\left(\theta_{1}^{*}, \theta_{2}^{*}\right) A$ for $\mathcal{Q}_{1}(1.2,1.8,0.5)$ with $h=\tau=$ $1 / 32$, respectively. These figures show that matrix $A$ maybe ill-conditioned while matrix $P^{-1}\left(\theta_{1}^{*}, \theta_{2}^{*}\right) A$ has tightly clustered eigenvalues around $(1,0)$, which implies that Method III can converge rapidly. Moreover, in Figure 3 we plot the error surfaces of Method III with $h=\tau=1 / 32$ for $\mathcal{Q}_{1}(1.2,1.8,0.5)$ at $t=0.5$ and $t=1$, respectively. This, again, testifies the effectiveness of Method III.

Example 5.2. Consider the following initial-boundary problem in the form (1.1) with

$$
\left\{\begin{array}{l}
a_{1}=0, \quad a_{2}=a_{3}=a_{4}=1, \quad a_{5}=-2 \cos \left(\frac{\alpha \pi}{2}\right), \quad a_{6}=-2 \cos \left(\frac{\beta \pi}{2}\right), \\
u_{0}(x, y)=0, \quad t_{0}=0, \quad T=1, \Omega=(0,1) \times(0,1),
\end{array}\right.
$$


Table 1: CPU times, global errors and convergence rates in time of Methods I-III with $h=1 / 60$ for $\mathcal{Q}_{1}(1.2,1.8, \gamma)$.

\begin{tabular}{|c|c|c|c|c|c|c|c|c|c|c|}
\hline \multirow[b]{2}{*}{$\gamma$} & \multirow[b]{2}{*}{$\tau$} & \multicolumn{3}{|c|}{ Method I } & \multicolumn{3}{|c|}{ Method II } & \multicolumn{3}{|c|}{ Method III } \\
\hline & & $\mathrm{CPU}$ & $\mathrm{E}(h, \tau)$ & Order $_{1}$ & $\mathrm{CPU}$ & $\mathrm{E}(h, \tau)$ & Order $_{1}$ & $\mathrm{CPU}$ & $\mathrm{E}(h, \tau)$ & Order $_{1}$ \\
\hline \multirow[t]{4}{*}{0.1} & $1 / 3$ & 166.77 & $1.5606 \mathrm{e}-2$ & - & 4.65 & $1.5606 \mathrm{e}-2$ & - & 1.92 & $1.5606 \mathrm{e}-2$ & - \\
\hline & $1 / 6$ & 326.77 & $8.4553 \mathrm{e}-3$ & 0.8841 & 7.47 & $8.4554 \mathrm{e}-3$ & 0.8841 & 3.49 & $8.4554 \mathrm{e}-3$ & 0.8841 \\
\hline & $1 / 12$ & 651.17 & $4.4454 \mathrm{e}^{-} 3$ & 0.9276 & 11.86 & $4.4454 \mathrm{e}-3$ & 0.9275 & 6.22 & $4.4455 \mathrm{e}-3$ & 0.9275 \\
\hline & $1 / 24$ & 1290.17 & $2.3263 \mathrm{e}-3$ & 0.9343 & 19.52 & $2.3264 \mathrm{e}-3$ & 0.9342 & 11.88 & $2.3264 \mathrm{e}-3$ & 0.9343 \\
\hline \multirow[t]{4}{*}{0.5} & $1 / 3$ & 166.73 & $1.8303 \mathrm{e}-2$ & - & 4.45 & $1.8303 \mathrm{e}-2$ & - & 1.91 & $1.8303 \mathrm{e}-2$ & - \\
\hline & $1 / 6$ & 324.59 & $9.2990 \mathrm{e}-3$ & 0.9770 & 6.97 & $9.2990 \mathrm{e}-3$ & 0.9770 & 3.40 & $9.2991 \mathrm{e}-3$ & 0.9770 \\
\hline & $1 / 12$ & 648.00 & $4.7046 \mathrm{e}-3$ & 0.9830 & 11.35 & $4.7046 \mathrm{e}-3$ & 0.9830 & 6.28 & $4.7047 \mathrm{e}-3$ & 0.9830 \\
\hline & $1 / 24$ & 1283.12 & $2.3766 \mathrm{e}-3$ & 0.9852 & 18.68 & $2.3767 \mathrm{e}-3$ & 0.9851 & 11.89 & $2.3767 \mathrm{e}-3$ & 0.9852 \\
\hline \multirow[t]{4}{*}{0.9} & $1 / 3$ & 164.28 & $2.6212 \mathrm{e}-2$ & - & 4.28 & $2.6212 \mathrm{e}-2$ & - & 1.91 & $2.6212 \mathrm{e}-2$ & - \\
\hline & $1 / 6$ & 324.77 & $1.3810 \mathrm{e}-2$ & 0.9245 & 6.53 & $1.3810 \mathrm{e}-2$ & 0.9245 & 3.37 & $1.3810 \mathrm{e}-2$ & 0.9245 \\
\hline & $1 / 12$ & 653.93 & $7.0496 \mathrm{e}-3$ & 0.9701 & 10.37 & $7.0496 \mathrm{e}-3$ & 0.9701 & 6.15 & $7.0497 \mathrm{e}-3$ & 0.9701 \\
\hline & $1 / 24$ & 1288.85 & $3.5514 \mathrm{e}-3$ & 0.9891 & 17.39 & $3.5516 \mathrm{e}-3$ & 0.9891 & 11.84 & $3.5515 \mathrm{e}-3$ & 0.9892 \\
\hline
\end{tabular}

Table 2: CPU times, global errors and convergence rates in space of Methods I-III with $\tau=1 / 1000$ for $\mathcal{Q}_{1}(\alpha, \beta, 0.5)$.

\begin{tabular}{|c|c|c|c|c|c|c|c|c|c|c|}
\hline \multirow[b]{2}{*}{$(\alpha, \beta)$} & \multirow[b]{2}{*}{$h$} & \multicolumn{3}{|c|}{ Method I } & \multicolumn{3}{|c|}{ Method II } & \multicolumn{3}{|c|}{ Method III } \\
\hline & & $\mathrm{CPU}$ & $\mathrm{E}(h, \tau)$ & $\mathrm{Order}_{2}$ & $\mathrm{CPU}$ & $\mathrm{E}(h, \tau)$ & $\mathrm{Order}_{2}$ & $\mathrm{CPU}$ & $\mathrm{E}(h, \tau)$ & $\mathrm{Order}_{2}$ \\
\hline \multirow[t]{4}{*}{$(1.2,1.8)$} & $1 / 3$ & 1.66 & $5.5429 \mathrm{e}-2$ & - & 9.99 & $5.5426 \mathrm{e}-2$ & - & 1.77 & $5.5429 \mathrm{e}-2$ & - \\
\hline & $1 / 6$ & 5.48 & $1.3788 \mathrm{e}-2$ & 2.0072 & 13.87 & $1.3784 \mathrm{e}-2$ & 2.0075 & 5.28 & $1.3787 \mathrm{e}-2$ & 2.0073 \\
\hline & $1 / 12$ & 25.46 & $3.4115 \mathrm{e}-3$ & 2.0149 & 31.64 & $3.4083 \mathrm{e}-3$ & 2.0159 & 18.75 & $3.4114 \mathrm{e}^{-}-3$ & 2.0149 \\
\hline & $1 / 24$ & 419.10 & $8.6726 \mathrm{e}-4$ & 1.9759 & 99.52 & $8.6319 \mathrm{e}-4$ & 1.9813 & 73.17 & $8.6761 \mathrm{e}-4$ & 1.9752 \\
\hline \multirow[t]{4}{*}{$(1.4,1.6)$} & $1 / 3$ & 1.68 & $5.4782 \mathrm{e}-2$ & - & 10.09 & $5.4781 \mathrm{e}-2$ & - & 1.78 & $5.4782 \mathrm{e}-2$ & - \\
\hline & $1 / 6$ & 5.52 & $1.3364 \mathrm{e}-2$ & 2.0353 & 14.14 & $1.3363 \mathrm{e}-2$ & 2.0354 & 5.27 & $1.3364 \mathrm{e}-2$ & 2.0353 \\
\hline & $1 / 12$ & 25.17 & $3.2843 \mathrm{e}-3$ & 2.0248 & 31.76 & $3.2826 \mathrm{e}-3$ & 2.0254 & 18.81 & $3.2824 \mathrm{e}-3$ & 2.0255 \\
\hline & $1 / 24$ & 413.83 & $8.3716 \mathrm{e}-4$ & 1.9720 & 99.77 & $8.3520 \mathrm{e}-4$ & 1.9747 & 72.87 & $8.3573 \mathrm{e}-4$ & 1.9736 \\
\hline \multirow[t]{4}{*}{$(1.5,1.5)$} & $1 / 3$ & 1.68 & $5.4686 \mathrm{e}-2$ & - & 9.99 & $5.4687 \mathrm{e}-2$ & - & 1.79 & $5.4686 \mathrm{e}-2$ & - \\
\hline & $1 / 6$ & 5.51 & $1.3308 \mathrm{e}-2$ & 2.0389 & 14.04 & $1.3307 \mathrm{e}-2$ & 2.0390 & 5.23 & $1.3306 \mathrm{e}-2$ & 2.0391 \\
\hline & $1 / 12$ & 25.68 & $3.2689 \mathrm{e}-3$ & 2.0254 & 31.54 & $3.2681 \mathrm{e}-3$ & 2.0257 & 18.60 & $3.2668 \mathrm{e}-3$ & 2.0262 \\
\hline & $1 / 24$ & 429.34 & $8.3402 \mathrm{e}-4$ & 1.9707 & 98.73 & $8.3104 \mathrm{e}-4$ & 1.9755 & 72.55 & $8.3275 \mathrm{e}-4$ & 1.9719 \\
\hline
\end{tabular}




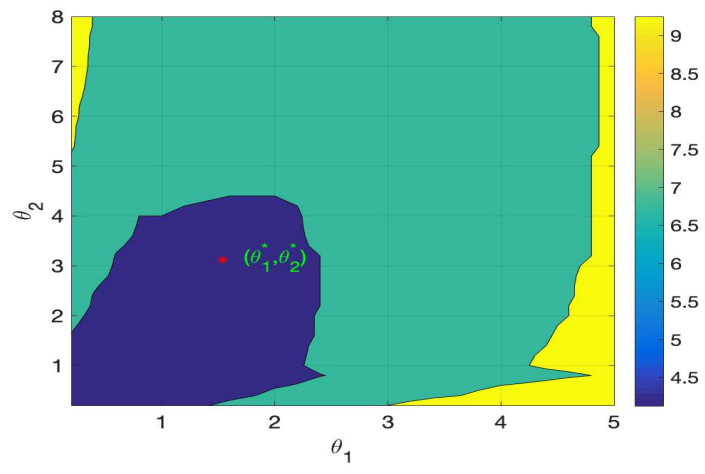

(a)

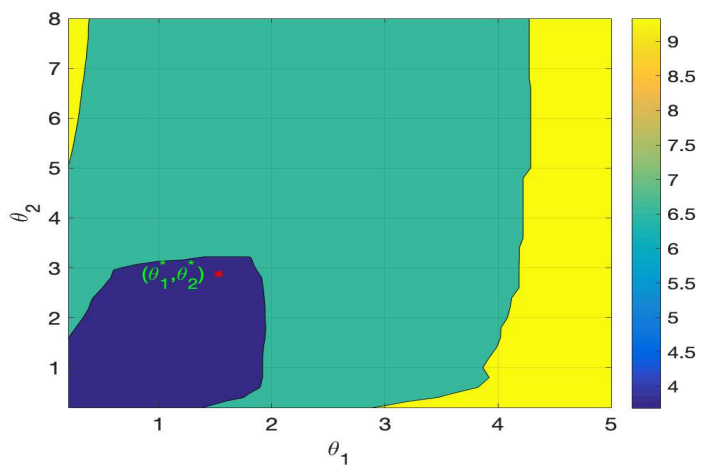

(b)

Figure 1: (a) Iteration numbers versus parameters $\theta_{1}, \theta_{2}$ for $\mathcal{Q}_{1}(1.2,1.8,0.5)$ with $h=\tau=1 / 16$; (b) Iteration numbers versus parameters $\theta_{1}, \theta_{2}$ for $\mathcal{Q}_{1}(1.2,1.8,0.5)$ with $h=\tau=1 / 32$.

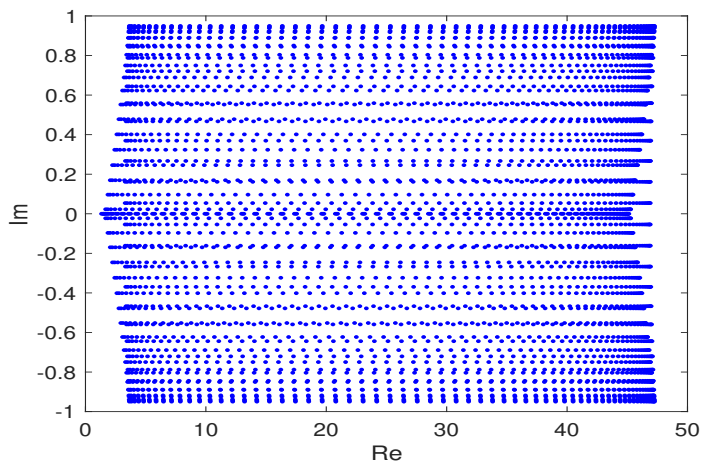

(a)

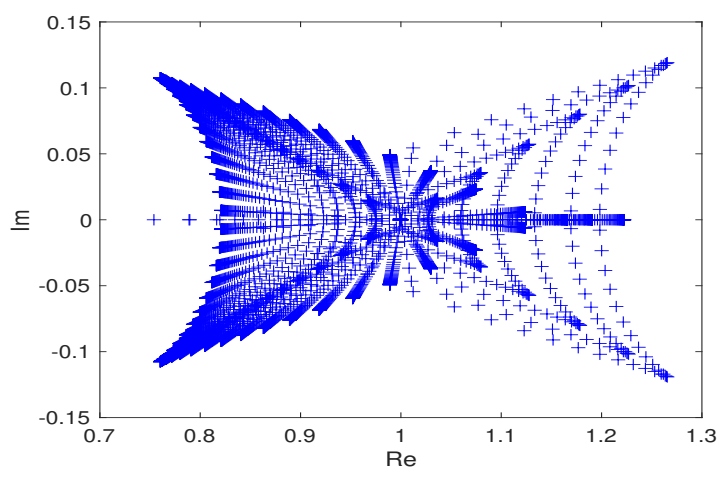

(b)

Figure 2: (a) The spectrum of matrix $A$ for $\mathcal{Q}_{1}(1.2,1.8,0.5)$ with $h=\tau=1 / 32$; (b) The spectrum of matrix $P^{-1}\left(\theta_{1}^{*}, \theta_{2}^{*}\right) A$ for $\mathcal{Q}_{1}(1.2,1.8,0.5)$ with $h=\tau=1 / 32$.

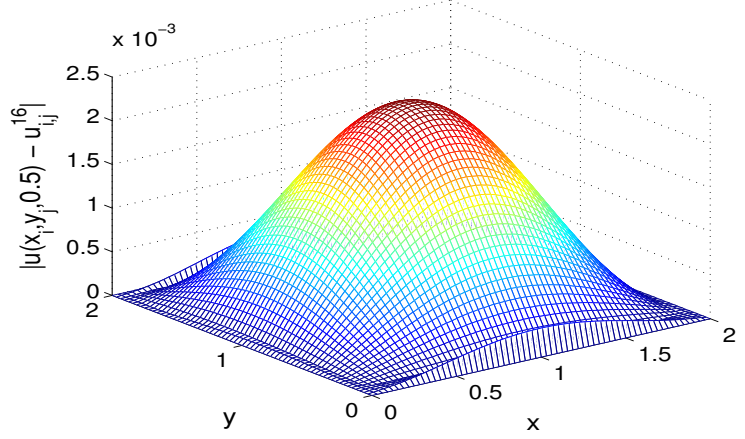

(a) $t=0.5$

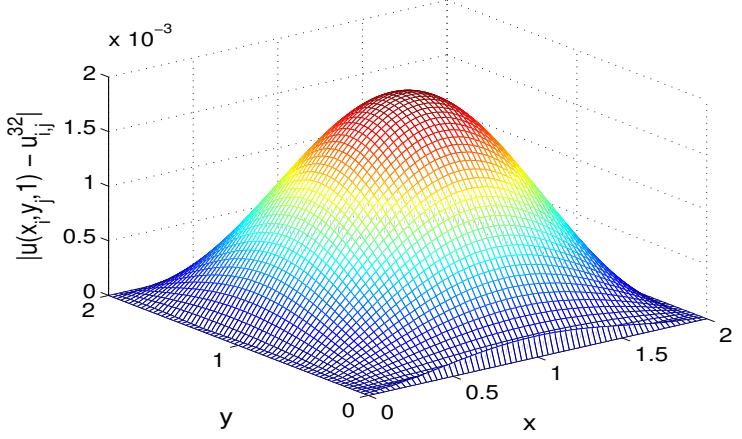

(b) $t=1$

Figure 3: Error surfaces of Method III with $h=\tau=1 / 32$ for $\mathcal{Q}_{1}(1.2,1.8,0.5)$ at $t=0.5$ and $t=1$, respectively. 
and source function $f(x, y, t)$ being assigned such that the problems have a common exact solution $u(x, y, t)=$ $2^{16}[t x(1-x) y(1-y)]^{4}$. For convenience, we write the discrete systems (4.1) corresponding to the above problems as $\mathcal{Q}_{2}(\alpha, \beta, \gamma)$.

Taking $h=1 /\left\lfloor 2(1 / \tau)^{\frac{2-\gamma}{2}}\right\rfloor$ and $\tau=1 / 8,1 / 16,1 / 32,1 / 64($ resp. $h=1 / 8,1 / 16,1 / 32,1 / 64$ and $\tau=1 / 500)$, then applying Methods I-III to the discrete systems $\mathcal{Q}_{2}(1.2,1.8, \gamma)$ with $\gamma=0.1,0.5,0.9$ (resp. $\mathcal{Q}_{2}(\alpha, \beta, 0.5)$ with $(\alpha, \beta)=(1.2,1.8),(1.4,1.6),(1.5,1.5))$, the CPU times (in second), global errors and convergence rates in time (resp. in space) are listed in Table 3 (resp. Table 4). From Tables 3-4, we can see that the three methods almost have the same accuracy under the same spatial and temporal stepsizes and reach the theoretical accuracy shown in Theorem 3.3. By comparing the CPU times, we see that the computational efficiency of Method III is optimal among the three methods.

In Figure 4 (a) (resp. Figure 4 (b)), we plot the average iteration numbers per time-step versus the parameters $\theta_{1}, \theta_{2}$ of Method III for $\mathcal{Q}_{2}(1.2,1.8,0.5)$ with $h=\tau=1 / 16$ (resp. $h=\tau=1 / 32$ ). From Figure 4 , it can be seen that there is a wide range of the parameters $\theta_{1}, \theta_{2}$ (including $\theta_{1}^{*}, \theta_{2}^{*}$ ) where the convergence of Method III is optimal. This also indicates that the selection of parameters $\theta_{1}^{*}$ and $\theta_{2}^{*}$ in Method III is appropriate. In Figure 5, we display the spectrums of matrices $A$ and $P^{-1}\left(\theta_{1}^{*}, \theta_{2}^{*}\right) A$ for $\mathcal{Q}_{2}(1.2,1.8,0.5)$ with $h=\tau=1 / 32$, respectively. This shows that matrix $A$ maybe ill-conditioned, while matrix $P^{-1}\left(\theta_{1}^{*}, \theta_{2}^{*}\right) A$ has tightly clustered eigenvalues around $(1,0)$, which implies that Method III can converge rapidly. Moreover, in Figure 6 we plot the error surfaces of Method III with $h=\tau=1 / 32$ for $\mathcal{Q}_{2}(1.2,1.8,0.5)$ at $t=0.5$ and $t=1$, respectively. This further confirms the effectiveness of Method III.

\begin{tabular}{|c|c|c|c|c|c|c|c|c|c|c|}
\hline \multirow[b]{2}{*}{$\gamma$} & \multirow[b]{2}{*}{$\tau$} & \multicolumn{3}{|c|}{ Method I } & \multicolumn{3}{|c|}{ Method II } & \multicolumn{3}{|c|}{ Method III } \\
\hline & & $\mathrm{CPU}$ & $\mathrm{E}(h, \tau)$ & Order $_{1}$ & CPU & $\mathrm{E}(h, \tau)$ & Order $_{1}$ & CPU & $\mathrm{E}(h, \tau)$ & Order $_{1}$ \\
\hline \multirow[t]{4}{*}{0.1} & $1 / 8$ & 0.11 & $5.0989 \mathrm{e}-3$ & - & 0.30 & $5.0989 \mathrm{e}-3$ & - & 0.15 & $5.0989 \mathrm{e}-3$ & - \\
\hline & $1 / 16$ & 0.60 & $1.6361 \mathrm{e}-3$ & 1.6399 & 0.53 & $1.6361 \mathrm{e}-3$ & 1.6399 & 0.37 & $1.6361 \mathrm{e}-3$ & 1.6399 \\
\hline & $1 / 32$ & 22.48 & $4.3751 \mathrm{e}-4$ & 1.9029 & 5.61 & $4.3751 \mathrm{e}-4$ & 1.9029 & 1.97 & $4.3751 \mathrm{e}-4$ & 1.9029 \\
\hline & $1 / 64$ & 1925.92 & $1.1785 \mathrm{e}-4$ & 1.8924 & 85.80 & $1.1785 \mathrm{e}-4$ & 1.8923 & 14.01 & $1.1785 \mathrm{e}-4$ & 1.8924 \\
\hline \multirow[t]{4}{*}{0.5} & $1 / 8$ & 0.05 & $1.3741 \mathrm{e}-2$ & - & 0.10 & $1.3741 \mathrm{e}-2$ & - & 0.04 & $1.3741 \mathrm{e}-2$ & - \\
\hline & $1 / 16$ & 0.15 & $5.1860 \mathrm{e}-3$ & 1.4058 & 0.24 & $5.1860 \mathrm{e}-3$ & 1.4058 & 0.13 & $5.1860 \mathrm{e}-3$ & 1.4058 \\
\hline & $1 / 32$ & 1.11 & $1.6794 \mathrm{e}-3$ & 1.6267 & 1.06 & $1.6794 \mathrm{e}-3$ & 1.6267 & 0.55 & $1.6794 \mathrm{e}-3$ & 1.6267 \\
\hline & $1 / 64$ & 21.26 & $6.1878 \mathrm{e}-4$ & 1.4404 & 5.81 & $6.1879 \mathrm{e}-4$ & 1.4404 & 2.74 & $6.1878 \mathrm{e}-4$ & 1.4404 \\
\hline \multirow[t]{4}{*}{0.9} & $1 / 8$ & 0.03 & $2.3876 \mathrm{e}-2$ & - & 0.09 & $2.3876 \mathrm{e}-2$ & - & 0.03 & $2.3876 \mathrm{e}-2$ & - \\
\hline & $1 / 16$ & 0.07 & $1.4304 \mathrm{e}-2$ & 0.7391 & 0.16 & $1.4304 \mathrm{e}-2$ & 0.7391 & 0.06 & $1.4304 \mathrm{e}-2$ & 0.7391 \\
\hline & $1 / 32$ & 0.21 & $7.0726 \mathrm{e}-3$ & 1.0161 & 0.34 & $7.0727 \mathrm{e}-3$ & 1.0161 & 0.19 & $7.0726 \mathrm{e}-3$ & 1.0161 \\
\hline & $1 / 64$ & 0.78 & $3.4066 \mathrm{e}-3$ & 1.0539 & 1.02 & $3.4066 \mathrm{e}-3$ & 1.0539 & 0.57 & $3.4066 \mathrm{e}-3$ & 1.0539 \\
\hline
\end{tabular}


Table 4: CPU times, global errors and convergence rates in space of Methods I-III with $\tau=1 / 500$ for $\mathcal{Q}_{2}(\alpha, \beta, 0.5)$.

\begin{tabular}{|c|c|c|c|c|c|c|c|c|c|c|}
\hline \multirow[b]{2}{*}{$(\alpha, \beta)$} & \multirow[b]{2}{*}{$h$} & \multicolumn{3}{|c|}{ Method I } & \multicolumn{3}{|c|}{ Method II } & \multicolumn{3}{|c|}{ Method III } \\
\hline & & $\mathrm{CPU}$ & $\mathrm{E}(h, \tau)$ & Order $_{2}$ & $\mathrm{CPU}$ & $\mathrm{E}(h, \tau)$ & Order $_{2}$ & $\mathrm{CPU}$ & $\mathrm{E}(h, \tau)$ & Order $_{2}$ \\
\hline \multirow{4}{*}{$(1.2,1.8)$} & $1 / 8$ & 0.88 & $2.1360 \mathrm{e}-2$ & - & 3.54 & $2.1360 \mathrm{e}-2$ & - & 0.97 & $2.1360 \mathrm{e}-2$ & - \\
\hline & $1 / 16$ & 3.40 & $4.9726 \mathrm{e}-3$ & 2.1028 & 5.37 & $4.9726 \mathrm{e}-3$ & 2.1028 & 2.83 & $4.9726 \mathrm{e}-3$ & 2.1028 \\
\hline & $1 / 32$ & 38.68 & $1.2196 \mathrm{e}-3$ & 2.0276 & 17.26 & $1.2196 \mathrm{e}-3$ & 2.0276 & 10.48 & $1.2196 \mathrm{e}-3$ & 2.0276 \\
\hline & $1 / 64$ & 965.18 & $3.0426 \mathrm{e}-4$ & 2.0030 & 96.55 & $3.0426 \mathrm{e}-4$ & 2.0030 & 41.67 & $3.0426 \mathrm{e}-4$ & 2.0030 \\
\hline \multirow[t]{4}{*}{$(1.4,1.6)$} & $1 / 8$ & 0.85 & $2.6132 \mathrm{e}-2$ & - & 3.73 & $2.6132 \mathrm{e}-2$ & - & 0.95 & $2.6132 \mathrm{e}-2$ & - \\
\hline & $1 / 16$ & 3.68 & $5.9927 \mathrm{e}-3$ & 2.1245 & 5.79 & $5.9927 \mathrm{e}-3$ & 2.1245 & 2.80 & $5.9927 \mathrm{e}-3$ & 2.1245 \\
\hline & $1 / 32$ & 28.86 & $1.4619 \mathrm{e}-3$ & 2.0354 & 15.71 & $1.4619 \mathrm{e}-3$ & 2.0354 & 10.68 & $1.4619 \mathrm{e}-3$ & 2.0354 \\
\hline & $1 / 64$ & 990.25 & $3.6408 \mathrm{e}-4$ & 2.0055 & 64.48 & $3.6409 \mathrm{e}-4$ & 2.0054 & 42.07 & $3.6409 \mathrm{e}-4$ & 2.0054 \\
\hline \multirow[t]{4}{*}{$(1.5,1.5)$} & $1 / 8$ & 0.89 & $2.6898 \mathrm{e}-2$ & - & 3.61 & $2.6898 \mathrm{e}-2$ & - & 0.99 & $2.6898 \mathrm{e}-2$ & - \\
\hline & $1 / 16$ & 3.66 & $6.1509 \mathrm{e}-3$ & 2.1286 & 5.80 & $6.1509 \mathrm{e}-3$ & 2.1286 & 2.83 & $6.1509 \mathrm{e}-3$ & 2.1286 \\
\hline & $1 / 32$ & 27.93 & $1.4990 \mathrm{e}-3$ & 2.0368 & 15.37 & $1.4990 \mathrm{e}-3$ & 2.0368 & 10.64 & $1.4990 \mathrm{e}-3$ & 2.0368 \\
\hline & $1 / 64$ & 1029.21 & $3.7322 \mathrm{e}-4$ & 2.0059 & 60.23 & $3.7323 \mathrm{e}-4$ & 2.0059 & 42.52 & $3.7322 \mathrm{e}-4$ & 2.0059 \\
\hline
\end{tabular}

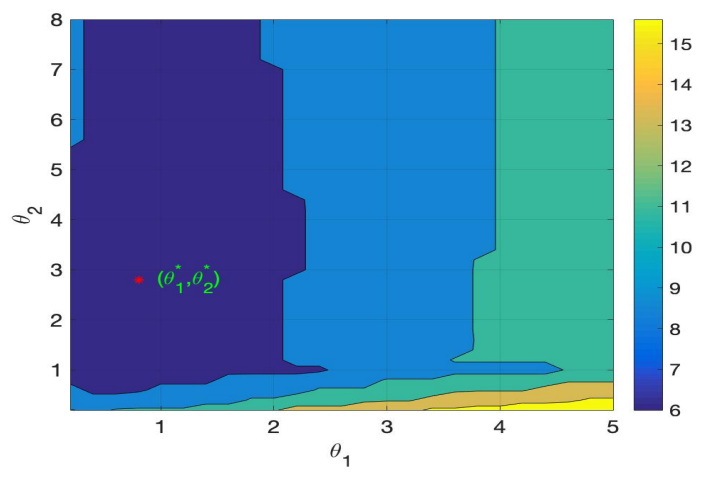

(a)

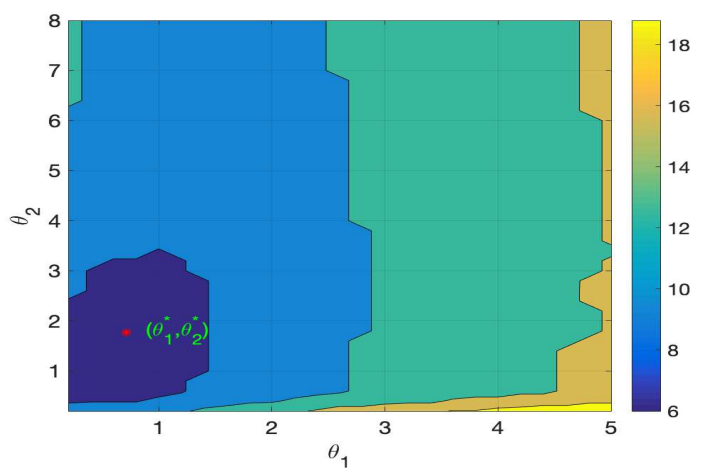

(b)

Figure 4: (a) Iteration numbers versus parameters $\theta_{1}, \theta_{2}$ for $\mathcal{Q}_{2}(1.2,1.8,0.5)$ with $h=\tau=1 / 16$; (b) Iteration numbers versus parameters $\theta_{1}, \theta_{2}$ for $\mathcal{Q}_{2}(1.2,1.8,0.5)$ with $h=\tau=1 / 32$.

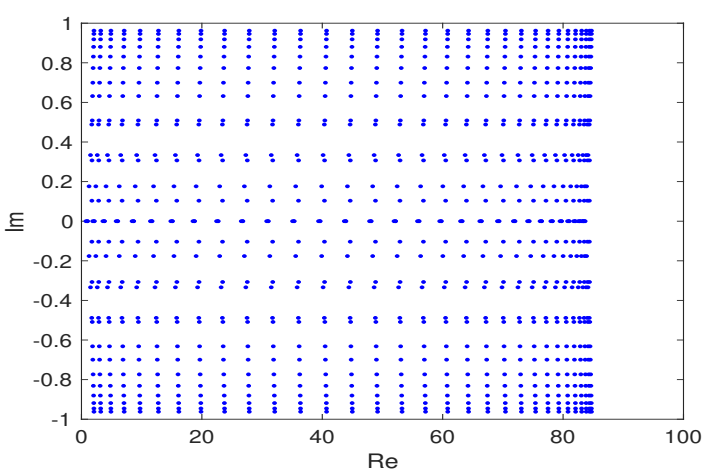

(a)

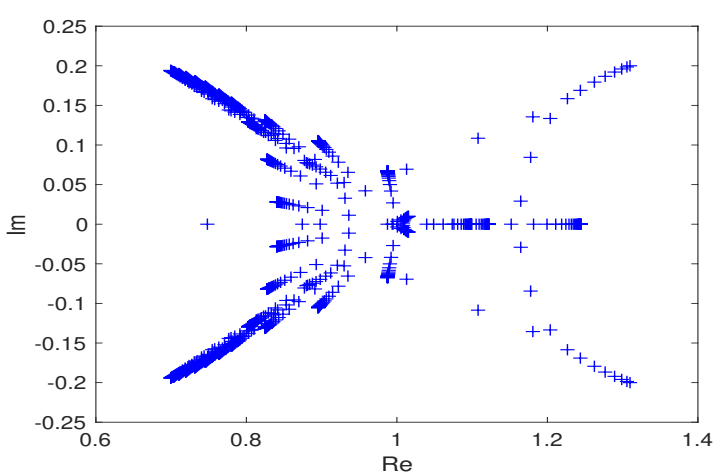

(b)

Figure 5: (a) The spectrum of matrix $A$ for $\mathcal{Q}_{2}(1.2,1.8,0.5)$ with $h=\tau=1 / 32$; (b) The spectrum of matrix $P^{-1}\left(\theta_{1}^{*}, \theta_{2}^{*}\right) A$ for $\mathcal{Q}_{2}(1.2,1.8,0.5)$ with $h=\tau=1 / 32$. 


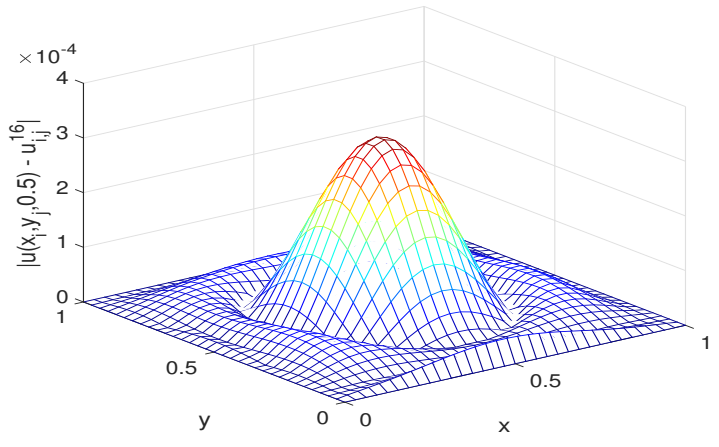

(a) $t=0.5$

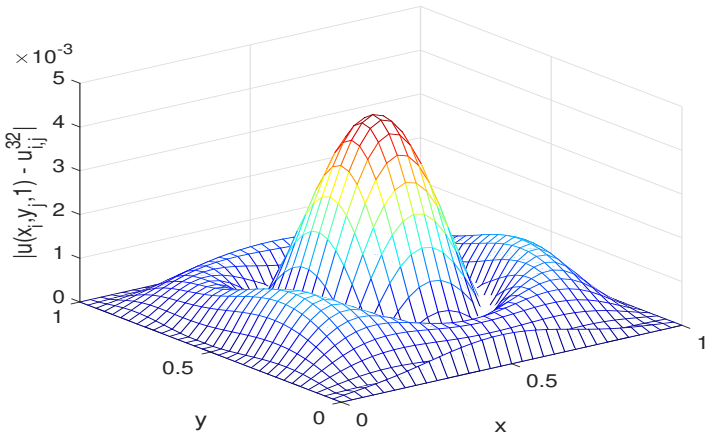

(b) $t=1$

Figure 6: Error surfaces of Method III with $h=\tau=1 / 32$ for $\mathcal{Q}_{2}(1.2,1.8,0.5)$ at $t=0.5$ and $t=1$, respectively.

\section{CRediT author statement}

Yongtao Zhou: Conceptualization, Methodology, Software, Validation, Formal analysis, Investigation, Resources, Data Curation, Writing-Original draft preparation, Visualization. Chengjian Zhang: Conceptualization, Methodology, Validation, Formal analysis, Investigation, Resources, Writing-Review \& Editing, Visualization, Supervision, Project administration, Funding acquisition. Luigi Brugnano: Conceptualization, Methodology, Validation, Formal analysis, Investigation, Writing-Review \& Editing.

\section{References}

[1] W. Deng, B. Li, W. Tian, P. Zhang, Boundary problems for the fractional and tempered fractional operators, Multiscale Model. Simul. 16 (2018) 125-149.

[2] F. Liu, V.V. Anh, I. Turner, P. Zhuang, Time fractional advection dispersion equation, J. Appl. Math. Comput. 13 (2003) 233-245.

[3] F. Liu, P. Zhuang, K. Burrage, Numerical methods and analysis for a class of fractional advection-dispersion models, Comput. Math. Appl. 64 (2012) 2990-3007.

[4] F. Liu, M.M. Meerschaert, R.J. McGough, P. Zhuang, Q. Liu, Numerical methods for solving the multi-term time-fractional wave-diffusion equation, Fract. Calc. Appl. Anal. 16 (2013) 9-25.

[5] R. Schumer, D.A. Benson, M.M. Meerschaert, B. Baeumer, Fractal mobile/immobile solute transport, Water Resour. Res. 39 (2003) 1296-1307.

[6] Y. Zhang, D.A. Benson, D.M. Reeves, Time and space nonlocalities underlying fractional-derivative models: Distinction and literature review of field applications, Adv. Water Resour. 32 (2009) 561-581.

[7] M.M. Meerschaert, H.P. Scheffler, Limit theorems for continuous time random walks with infinite mean waiting times, J. Appl. Probab. 41 (2004) 623-638.

[8] P. Becker-Kern, M.M. Meerschaert, H.P. Scheffler, Limit theorems for coupled continuous time random walks, Ann. Probab. 32 (2004) 730-756.

[9] D.A. Benson, S.W. Wheatcraft, M.M. Meerschaert, The fractional-order governing equation of Lévy motion, Water Resour. Res. 36 (2000) 1413-1423.

[10] F. Liu, V.V. Anh, I. Turner, Numerical solution of the space fractional Fokker-Planck equation, J. Comput. Appl. Math. 166 (2004) 209-219.

[11] K. Oldham, J. Spanier, The Fractional Calculus: Theory and Applications of Differentiation and Integration to Arbitrary Order, Academic Press, New York, 1974.

[12] Z. Sun, X. Wu, A fully discrete difference scheme for a diffusion-wave system, Appl. Numer. Math. 56 (2006) $193-209$.

[13] D. Li, J. Wang, J. Zhang, Unconditionally convergent L1-Galerkin FEMs for nonlinear time-fractional Schrödinger equations, SIAM J. Sci. Comput. 39 (2017) 3067-3088.

[14] B. Jin, R. Lazarov, Z. Zhou, An analysis of the $L 1$ scheme for the subdiffusion equation with nonsmooth data, IMA J. Numer. Anal. 36 (2015) 197-221.

[15] M.M. Meerschaert, C. Tadjeran, Finite difference approximations for fractional advection-dispersion flow equations, J. Comput. Appl. Math. 172 (2004) 65-77.

[16] M.M. Meerschaert, C. Tadjeran, Finite difference approximations for two-sided space-fractional partial differential equations, Appl. Numer. Math. 56 (2006) 80-90. 
[17] Z. Hao, Z. Sun, W. Cao, A fourth-order approximation of fractional derivatives with its applications, J. Comput. Phys. 281 (2015) 787-805.

[18] W. Tian, H. Zhou, W. Deng, A class of second order difference approximation for solving space fractional diffusion equations, Math. Comput. 84 (2015) 1703-1727.

[19] M. Chen, W. Deng, Fourth order accurate scheme for the space fractional diffusion equations, SIAM J. Numer. Anal. 52 (2014) 1418-1438.

[20] E. Sousa, C. Li, A weighted finite difference method for the fractional diffusion equation based on the Riemann-Liouville derivative, Appl. Numer. Math. 90 (2011) 22-37.

[21] H. Wang, K. Wang, T. Sircar, A direct $\mathcal{O}\left(N \log _{2} N\right)$ finite difference method for fractional diffusion equations, J. Comput. Phys. 229 (2010) 8095-8104.

[22] H. Pang, H. Sun, Multigrid method for fractional diffusion equations, J. Comput. Phys. 231 (2012) 693-703.

[23] L. Chou, S. Lei, Fast ADI method for high dimensional fractional diffusion equations in conservative form with preconditioned strategy, Comput. Math. Appl. 73 (2017) 385-403.

[24] S. Lei, H. Sun, A circulant preconditioner for fractional diffusion equations, J. Comput. Phys. 242 (2013) 715-725.

[25] S. Lei, X. Chen, X. Zhang, Multilevel circulant preconditioner for high-dimensional fractional diffusion equations, East Asian J. Appl. Math. 6 (2016) 109-130.

[26] F. Lin, S. Yang, X. Jin, Preconditioned iterative methods for fractional diffusion equation, J. Comput. Phys. 256 (2014) 109-117.

[27] H. Chen, W. Lv, T. Zhang, A Kronecker product splitting preconditioner for two-dimensional space-fractional diffusion equations, J. Comput. Phys. 360 (2018) 1-14.

[28] Y. Zhou, C. Zhang, L. Brugnano, Preconditioned quasi-compact boundary value methods for space-fractional diffusion equations, Numer. Algorithms (2019) https://doi.org/10.1007/s11075-019-00773-z.

[29] Y. Saad, M.H. Schultz, GMRES: A generalized minimal residual algorithm for solving nonsymmetric linear systems, SIAM J. Sci. Stat. Comput. 7 (1986) 856-869.

[30] S. Vong, P. Lyu, X. Chen, S. Lei, High order finite difference method for time-space fractional differential equations with Caputo and Riemann-Liouville derivatives, Numer. Algorithms 72 (2016) 195-210.

[31] R.A. Horn, C.R. Johnson, Topics in Matrix Analysis, Cambridge University Press, Cambridge, 1991.

[32] M. Benzi, G.H. Golub, A preconditioner for generalized saddle point problems, SIAM J. Matrix Anal. Appl. 26 (2004) $20-41$. 